\title{
Real-Time Computing Cornerstones: A System Engineer's View
}

\author{
Michael W. Masters \\ Naval Surface Warfare Center Dahlgren Division, Dahlgren VA 22448
}

\begin{abstract}
Common attributes are found in both military and commercial real-time systems. The existence of these common threads suggests that there may be key quantitative system design considerations that undergird large scale, real-time system design. Identifying and quantifying these key aspects early in a program is important in insuring the success of a design. This paper suggests a candidate list of such computing properties, or cornerstones, by which every real-time system is defined and constrained.
\end{abstract}

\section{Common Threads of System Design}

Although capabilities, components and architectures differ dramatically, there are common threads and design factors that run through military systems, process control, telecommunications, commercial aviation, air traffic control, and space systems. For example, no matter how fast the processor, meeting critical timelines always seems to be the paramount concern. Communication among system components is almost always a bottleneck. Low latency is usually more important than high throughput, and the two are not reciprocals of each other. If the system goes down during its active duty cycle, something expensive and/or awful happens. And, no matter how carefully the system is designed, someone will want to change it in ways not anticipated by the original designers.

These common threads suggest that there may be fundamental principles involved, cornerstones as it were, that characterize and undergird the art and science of large scale, real-time system design. Identifying these attributes, defining the relationships between them and assigning quantitative allowances for them in the overall design early in a program is important in insuring the success of a design. Rear Admiral Wayne E. Meyer (retired), father of the U.S. Navy's AEGIS guided missile cruisers and destroyers, described the importance of establishing the metrics that drive system engineering early in a development program. [1] "You have to be able to define what's needed in pretty simple terms, and you have to work to that definition ... Every complex system must be reduced to a simple set of numbers. I believe that they are functional and operational numbers . . . and if you cannot do it I don't believe you can build it."

Early in the design of AEGIS, a highly complex, integrated multi-warfare system involving many computers and millions of lines of code, Meyer set quantitative targets for five key performance factors: firepower, coverage, reaction time, electromagnetic environment resistance, and continuous availability. "One thing we knew was that we had to achieve that set of numbers, and we spent a long time convincing ourselves that that was the complete set." These comerstones were, in fact, more than just numbers. Like simultaneous equations in multiple unknowns, the numbers had to exist in harmony with each other. "What equations are these? ... I call them the five partial non-linear differential equations. Now partial differential equations all have a common characteristic, and that is that they have no [closed] solution . .. So these [equations] require simultaneous solutions, not piecemeal solutions."

Similarly, successful design of real-time computer systems requires solving many simultaneous equations involving key computing attributes. A computer system exists to support and enable the operation of the larger system in which it is embedded. The values chosen for these computing attributes must be selected to support the accomplishment of the larger system's purpose. Thus computing cornerstones must be expressed in terms that relate to computing, not to the domain of any particular system. Here are some candidates.

\section{Computing Cornerstones}

Capacity. The quantitative measure of computing resources needed to perform required functions, including CPU cycles, memory, mass storage, etc. Also includes overhead associated with operating system services, communication protocols, $\mathrm{I} / \mathrm{O}$ drivers, etc.

Connectivity. Interface attributes, including numbers and types of connections, as well as performance characteristics of those connections, required to support all external and internal communication among system components. 
Control. Bounded, deterministic, quantifiable management of time, computing resources, sequence of computation, and response to external events.

Continuous Availability. The level of required availability, within a system's active duty cycle, of the computing resources neded to carry out the system's mission. Usually near 100 percent for real-time systems.

Correctness. The likelihood that a system will perform its mission as required. The absence of flaws of design and implementation that jeopardize mission performance.

Efficacy of Design. The set of those design metrics that contribute to the life cycle cost effectiveness of a system, including cost of purchase, cost of ownership, ease of change, and other engineering "-ilities" that characterize flexibility of design and insensitivity to change and growth.

\section{Description}

Capacity is perhaps the most obvious of these attributes. Anyone who has ever designed a large system has had to face the fact that computer resources are not unlimited. For large systems, the process of benchmarking candidate computers with realistic test programs, deciding the number of processors, and allocating computational tasks to those resources can make or break a development effort. While memory and disk storage are relatively less expensive today than in the past, they are nevertheless still important parameters in system design.

For systems with many components, communication connectivity and bandwidth are often the critical resources in the system. Older systems were often based on point-to-point connections, which provided dedicated bandwidth but limited the number of connections. Modern shared media networks, though suitable for non-real-time use, often impose unacceptable limits on latency and determinism for real-time users. Newer, switch-based networks appear to be superior, although much evaluation remains to be done. Note that this category includes backplanes and internal and external shared memory. Backplanes are shared resources too, and their bandwidth can easily be exceeded by placing too many demands on them.

Providing applications with control mechanisms is largely the domain of the operating system. It is crucial in determining whether or not a system can achieve its real-time performance requirements. Critical features include scheduling policies, priorities, preemption---including kernel preemption, use of timers, synchronization primitives, interrupt latencies, system services execution times, context switch times, existence of lightweight constructs such as threads, memory management efficiency, detection of software exceptions and hardware fault conditions, support for startup and restart, etc.

For most real-time systems, continuous availability is a requirement within some operational cycle. Significant down time for repair and replacement is not possible during that time. Thus, the system design must address availability in a fundamental way, usually by redundancy. For those systems where damage to the system may result from the performance of its mission, survivability becomes a consideration as well. These systems require fast and reliable fault detection, system state replication, and failover control mechanisms. Such mechanisms often are quite sophisticated, and they require much engineering time to design and test time to validate.

Correctness may seem to be less quantifiable, or at least less subject to before-the-fact engineering. And yet, correctness is a primary goal of the entire system engineering process, from requirements analysis through design review, code walkthrough, unit debug, system test, and formal certification. Problem resolution processes exist for almost all fielded real-time systems to insure continuing correctness. Even today, problem reports per unit of time is considered a measure of correctness. Use of formal methods for proof of correctness is gaining support, and it may well represent a viable solution for "engineering in" correctness in the future.

The cost side of efficacy of design is easy enough to understand. Economic considerations have a pervasive impact on every system, whether military or commercial. But choices made during initial design, choices that may have little impact on cost of purchase, can have a dramatic impact on the ease with which changes can be made later. This attribute goes by many names, e.g. flexibility, extensibility, scalability, etc. But these "-ilities" are all different views of the same metric: the ability to adapt the system to new situations without requiring that the original system be thrown away and a new one built from scratch. The open systems approach has arisen largely in response to the economic value of making change easy. While we may yet lack the science to quantify this attribute before the fact, it is real and vital nonetheless.

Any list is likely to provoke considerable discussion, pro and con. Nevertheless, the six computing attributes introduced herein are offered as a set of real-time computing comerstones, the quantitative characteristics of which must be established in advance and met during the engineering of any system. In designing a real-time system, these key computing attributes do not stand alone. They must be solved simultaneously.

\section{Reference}

[1] RADM Wayne E. Meyer, USN (ret.), address to First Annual Workshop on Engineering of Systems in the 2lst Century: Facing the Challenge, 28 June 1994. 